\title{
Multi-object spectroscopy of stars in the CoRoT fields
}

\section{Early-type stars in the CoRoT-fields IRa01, LRa01, LRa02 ${ }^{\star \star \star}$}

\author{
D. Sebastian ${ }^{1}$, E. W. Guenther ${ }^{1}$, V. Schaffenroth ${ }^{2}$, D. Gandolfi ${ }^{3}$, S. Geier ${ }^{2}$, U. Heber $^{2}$, M. Deleuil ${ }^{4}$, and C. Moutou ${ }^{4}$ \\ 1 Thüringer Landessternwarte Tautenburg, Sternwarte 5, 07778 Tautenburg, Germany \\ e-mail: sebastian@tls-tautenburg.de \\ 2 Dr. Karl Remeis-Observatory \& ECAP, Astronomical Institute, Friedrich-Alexander University Erlangen-Nuremberg, \\ Sternwartstr. 7, 96049 Bamberg, Germany \\ 3 Research and Scientific Support Department, European Space Agency, Keplerlaan 1, 2200AG Noordwijk, The Netherlands \\ ${ }^{4}$ Laboratoire d'Astrophysique de Marseille, 38 rue Frédéric Joliot-Curie, 13388 Marseille Cedex 13, France
}

Received 5 September 2011 / Accepted 21 February 2012

\begin{abstract}
Context. Observations of giant stars indicate that the frequency of giant planets is much higher for intermediate-mass stars than for solar-like stars. Up to now all known planets of giant stars orbit at relatively far distances from their host stars. It is not known whether intermediate-mass stars also had many close-in planets when they were on the main sequence, which were then engulfed when the star became a giant star. To understand the formation and evolution of planets it is therefore important to find out whether main-sequence stars of intermediate-mass have close-in planets or not.

Aims. A survey for transiting planets of intermediate-mass stars would be ideal to solve this question, because the detection of transiting planets is not affected by the rapid rotation of these stars. With CoRoT it is possible to detect transiting planets around stars up to a spectral type B4V. As a first step for an efficient survey we need to identify intermediate-mass stars in the CoRoT-fields, which can then be used as an input list.

Methods. To compile the input list we derived the spectral types of essentially all O, B and A stars down to 14.5 mag in the CoRoT fields IRa01, LRa01, LRa02 taken with the multi-object spectrograph AAOmega. We determined the spectral types by comparing the spectra with template spectra from a library.

Results. In total we identify $1856 \mathrm{~A}$ and B stars that have been observed with CoRoT. Using multiple spectra of these stars, we find that the accuracy of the resulting spectral classification is $1.61 \pm 0.14$ sub-classes for A and B stars.

Conclusions. Given the number of planets that have been detected in these fields amongst late-type stars, we estimate that there are one to four transiting planets of intermediate-mass stars waiting to be discovered. Our survey not only allows us to carry out a dedicated planet search programme but is also essential for any types of studies of the light curves of early-type stars in the CoRoT database. We also show that it would be possible to extend the survey to all fields that CoRoT has observed using photometrically determined spectral types.
\end{abstract}

Key words. techniques: spectroscopic - planets and satellites: formation - surveys - stars: early-type - planets and satellites: detection

\section{Introduction}

\subsection{The frequency of planets orbiting A-type stars}

At present, more than 400 planets orbiting low-mass mainsequence stars (G-type stars and later) have been detected indirectly by means of precise radial-velocity (RV) surveys. Moreover transit search programmes discovered more than 100 transiting planets (The Extrasolar Planets Encyclopaedia ${ }^{1}$ ). These data provide detailed information about the properties of planets orbiting main-sequence stars with $M \leq$ 1.2 $M_{\odot}$. However, our knowledge about the planets, orbiting intermediate-mass stars (A and B-type stars) is still very limited. The reason is that it is difficult to detect planets of

\footnotetext{
* Based on observations obtained Anglo-Australian Telescope in programme 07B/040 and 08B/003.

$\star \star$ Table 1 is only available at the CDS via anonymous ftp to cdsarc.u-strasbg.fr (130.79.128.5) or via

http://cdsarc.u-strasbg.fr/viz-bin/qcat?]/A+A/541/A34

${ }^{1}$ http://www . exoplanet.eu, 2 August 2011.
}

main-sequence stars with masses larger than $\left(1.2 M_{\odot}\right)$ using the RV-technique. This is because most of these stars rotate rapidly, which significantly reduces the accuracy of the RV-measurements because of the line broadening.

The most important source of information about planets of intermediate-mass stars are RV-surveys of giant stars. About 30 planets orbiting giant stars have been discovered, many of them orbiting stars that were more massive than the Sun when they were on the main sequence. All groups studying these stars come to the same conclusion: intermediate-mass stars are more likely to have planets than solar-like stars (G-type stars). For example, Johnson et al. (2010a,b) conclude that the frequency of planets orbiting stars in the mass range between 1.5 and $3 M_{\odot}$ is a factor of 2 to 4 higher than for stars of $1.0 M_{\odot}$, which agrees well with the theoretical expectations (Kennedy \& Kenyon 2008).

The high frequency of planets orbiting giant stars is particularly surprising, because all known planets of giant stars have long orbital periods (semi-major axis between 0.5 and $2.6 \mathrm{AU}$ ), and are relatively massive with a median of $5.3 M_{\text {Jup }}$ (The Extrasolar Planets Encyclopaedia). It is interesting to note that 
the lack of close-in planets orbiting giant stars is in relative terms even more extreme than for solar-like stars. Planets of giant stars have distances $>8 R_{*}$, whereas planets of solar-like stars have distances that can be much closer than $3 R_{*}$. As shown by Bowler et al. (2010), the differences between the properties of planets orbiting giant and low-mass main-sequence stars is not an artefact of the sample size, but the two populations are different. Alibert et al. (2011) modelled the properties of the planetary population for different stellar masses from planet-formation theories. These authors predicted that stars with $2 M_{\odot}$ should have massive, close-in planets, if the disc-mass does depend on the stellar mass. If it does not, Alibert and collaborators do not expect close-in planets. Moreover, they predict that the properties of close-in planets orbiting intermediate-mass stars depend much more on the properties of the disc than do those planets that orbit solar-like stars.

The crucial question is therefore, what caused the observed lack of planets orbiting giant stars within $0.5 \mathrm{AU}$. There are three possible scenarios. The first hypothesis is that the lifetime of the gaseous disc of intermediate-mass stars is so short that there is not enough time for the planets to migrate inwards (Currie 2009). A second hypothesis is that the planets migrate outwards during the evolution of the star. It is true that planets move outwards because of the mass-loss of the star, but this effect is not strong enough to explain the complete lack of planets within $0.5 \mathrm{AU}$. A third possibility is that intermediate-mass stars have close-in planets when they are on the main sequence but that these are engulfed when the star becomes a giant star (Sato et al. 2008). Calculations by Villaver \& Livio (2009) show that tidal interaction can lead to the engulfment of close-in planets by evolved stars. Because the engulfment of planets may affect the evolution of stars, it would be important to establish whether this process is common, or rare. Which of the three possibilities is correct can only be solved by a dedicated survey for close-in planets orbiting main-sequence stars of intermediate-mass.

There are indeed several planets known that orbit intermediate-mass stars before they become giant stars. Using direct imaging techniques, planets of the A stars $\beta$ Pic (Lagrange et al. 2009), HR 8799 (Marois et al. 2008), and Fomalhaut have been detected (Kalas et al. 2008). Because the time for the formation of the core scales with the orbital period and all of these planets have orbital periods of more than 15 years, the very existence of these planets tells us that there must have been also enough time for close-in planets to form. However, these long-period planets are not suitable to prove the predictions for close-in planets.

Johnson et al. (2010b) reported the discovery of a giant planet orbiting HD 102956 at a close distance of 0.081 AU. The planet was detected by precise RV-measurements of the $1.68 M_{\odot}$-star, which is classified as an A-sub-giant star.

The only known transiting planet orbiting an A-type star is WASP-33b. It is a very close-in planet with an orbital distance of $0.026 \mathrm{AU}$ (Cameron et al. 2010). It is interesting to note, that this planet is currently the hottest planet known (Smith et al. 2011). Studying these planets helps us to understand whether evaporation plays an important role in the evolution of planets. Because the amount of evaporation depends on the density of the planet and the amount of radiation that the planet receives (Lammer et al. 2009), we need a sample of close-in planets for which these parameters are determined. Another interesting aspect is that Herrero et al. (2011) detected $\delta$ Sct-like oscillations that might be induced by the planet. If all close-in planets of A stars would induce oscillations, it would be easy to understand why only one has been found. It is also interesting to note that there

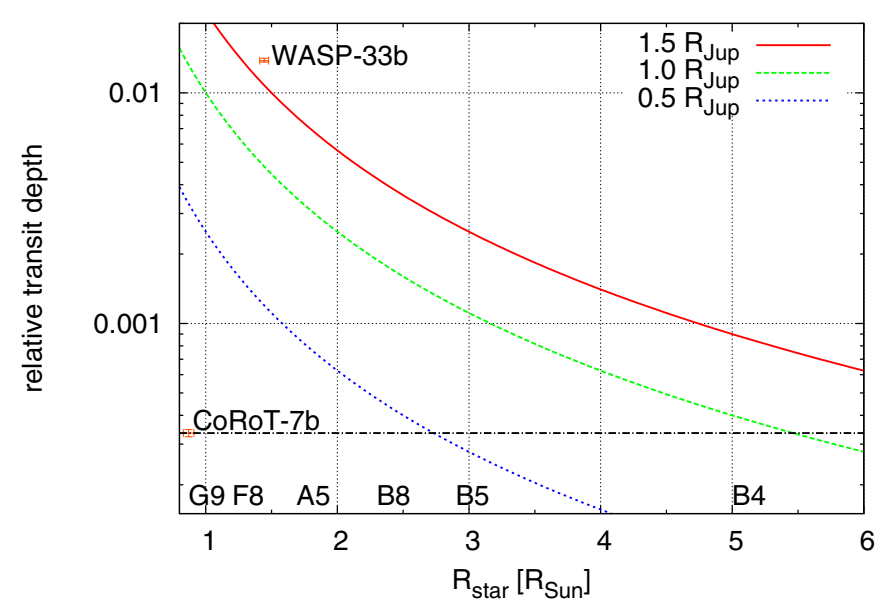

Fig. 1. Detectability of planets of $1.5,1.0$, and $0.5 R_{\text {Jup }}$ orbiting stars of different spectral types.

are transit candidates of early-type stars published by the CoRoT (Carpano et al. 2009) and Kepler space-missions (Borucki et al. 2011). Since the spectral types of these candidates are not well known, none of these candidates were confirmed as planets of an intermediate-mass star so far. These discoveries show the importance of a dedicated search programme for detecting planets orbiting A-type stars.

\subsection{A new survey for transiting planets of $A$ and $B$ stars}

As outlined above, transit search programmes are ideal for finding close-in planets of early-type stars. Space-based transit search programmes have several advantages over ground-based search programmes: not only is the obtained photometric accuracy much higher, the excellent time-coverage also allows one to remove the oscillations more efficiently. The removal of stellar oscillations would be particularly important if it turned out that close-in planets can induce $\delta$ Sct-like oscillations. Last not least, a long time-series allows one to average many transits, which makes the detection of shallow transits easier.

We used data obtained with the CoRoT (COnvection ROtation \& planetary Transits) satellite (Auvergne et al. 2009). CoRoT has detected a transiting planet with a relative transit depth of $3.4 \times 10^{-4}$ orbiting a star of $11.7 \mathrm{mag}$ (Léger et al. 2009). This means that CoRoT has the capability to detect a transiting planet with the size of Jupiter orbiting a B4V star (Fig. 1). It might be possible to detect planets of A stars down to $14.5 \mathrm{mag}$. This compares favourably with ground-based observations, which achieve an accuracy of only about 0.01 . For the long runs, the fields were observed continuously by CoRoT for up to 150 days. In the normal mode, the time-sampling is $8.5 \mathrm{~min}$.

We first identified suitable targets for our transit search programme and then carried out a dedicated analysis of the light curves. We selected the first three fields that CoRoT observed in the so-called anti-centre "eye": IRa01 (RA $100.9^{\circ}$ to $102.6^{\circ}$, Dec $-0.5^{\circ}$ to $-3.3^{\circ}$ ), LRa01 (RA $100.1^{\circ}$ to $101.5^{\circ}$, Dec $1.1^{\circ}$ to $-1.6^{\circ}$ ), and $\mathrm{LRa} 02$ (RA $101.9^{\circ}$ to $103.5^{\circ}$, Dec $-3.0^{\circ}$ to $-5.9^{\circ}$ ). Prior to the launch of the satellite, these fields were observed in $B, V, R$, and $I$ (Deleuil et al. 2006). These observations were cross-correlated with 2 MASS $J, H, K_{\mathrm{s}}$ magnitudes (Cutri et al. 2003) and used to perform a dwarf-giant discrimination and spectral classification. The photometric measurements in both 
sets of filters and the spectral classification are available through the database EXODAT ${ }^{2}$ (Deleuil et al. 2009)

However, we did not apply any pre-selection criteria but analysed all stars down to $M_{V}=14.5$ spectroscopically. The observation and spectroscopic analysis of all stars on the one hand provides us with a suitable target list and on the other hand gives the opportunity to verify the accuracy, if we were to use a target list compiled from photometric data (see Sect. 6).

Our survey for spectral classification of stars in the CoRoTfields IRa01, LRa01, and LRa02 divided into two parts. One contains a catalogue of all $\mathrm{O}, \mathrm{B}$, and A stars found in this survey. This catalogue is the input catalogue for a dedicated search programme for planets of intermediate-mass stars. The second part of the survey contains the catalogue of F, G, K and M stars (Guenther et al., in prep., Paper II), which aims at statistically comparing the spectral types of planet host stars with the sample of stars that have been observed. We excluded the early-type stars from Paper II, because no dedicated survey of planets of early-type stars has been carried out so far.

A survey for early-type stars in the CoRoT-fields is not only important for planet search programmes, but is also essential for any study of the CoRoT-light curves of early-type stars.

An example are the fields of most of the surveys for hotsubdwarf stars ( $\mathrm{sdO} / \mathrm{B})$, which are of special interest for close binary research and asteroseismology (for a review, see Heber 2009). These fields are arranged in a perpendicular direction to the Galactic plane. The spectroscopic identification of $\mathrm{O}$ - and B-type stars is the first step for identitying the as yet unknown population of hot-subdwarf stars situated in the Galatic plane. The analysis of high-precision sdB light curves obtained by the Kepler mission (e.g. Østensen et al. 2010) led to very interesting results and showed us that we can learn a lot about the properties of these objects by extending these analyses to the CoRoT-fields.

Another application of our survey are studies of rapidly oscillating Ap (roAp), or $\gamma$ Doradus stars. These objects have been identified in the Kepler field (e.g. Balona et al. 2011) but not yet in the CoRoT-fields.

Using the multi-object spectrograph FLAMES/GIRAFFE, Gazzano et al. (2010) have studied the stellar population in the CoRoT-fields LRa01, LRc01, and SRc01, but since there is only one A-star in that survey that we also observed (CoRoT 102677302), we compare the results of Gazzano and collaborators with our results in Paper II (Guenther et al., in prep.).

\section{Spectroscopic observations}

As part of the CoRoT follow-up observations, we observed stars in IRa01, LRa01, and LRa02 with the AAOmega multiobject spectrograph mounted on the AAT (Anglo-Australian Telescope/Australian Astronomical Telescope). The AAOmega multi-object-spectrograph is ideal for this purpose, because it has a field of view of 2 degrees, which perfectly matches the size of the CoRoT fields, which have a size of $1.8 \times 3.6$ squaredegrees (Saunders et al. 2004; Smith et al. 2004).

The data were obtained in two campaigns. The first campaign was carried out between 13-20 January 2008. Unfortunately, owing to the weather conditions, observations could only be carried out in the first two nights. The second campaign was carried out from 28 December 2008 to 4 January 2009 and observations were carried out during all eight nights.

\footnotetext{
2 The EXODAT database is continuously updated and available online: http://cesam.oamp.fr/exodat/
}

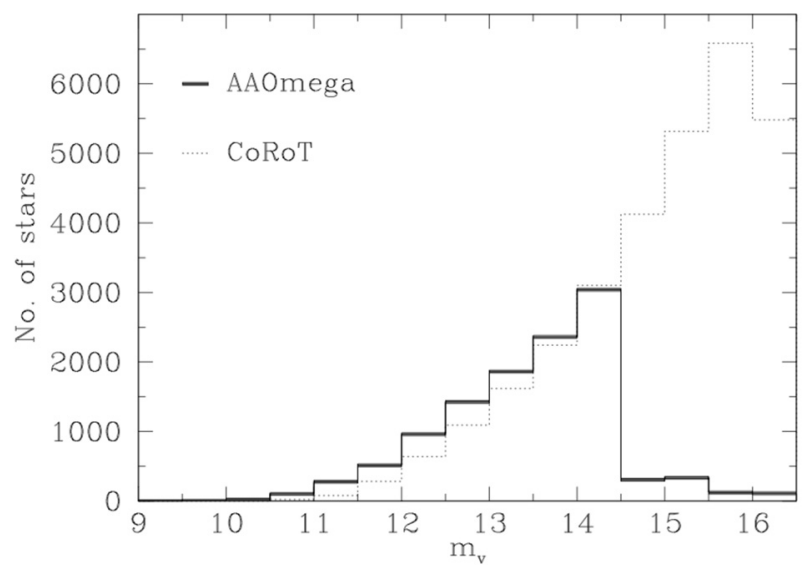

Fig. 2. Number of stars observed by CoRoT (dotted line) compared to the number of stars that we analysed for each brightness interval (solid line).

We used the AAT software tool "Configure" for our target allocation to find the optimum configuration of the fibres. In each setting we typically placed 350 fibres on target stars, and 25 on the sky background. We also added stars that are not observed by CoRoT to maximise the number of stars observed in each setting. To optimise the exposure time and to avoid cross-talk between the fibres, all stars observed in each setting have in general the same brightness within half a magnitude. We started our observations with the fields containing the brightest stars and subsequently used settings for increasingly fainter stars.

Figure 2 shows a histogram of the 31405 stars observed by CoRoT in the three fields and the 11466 stars observed and analysed here. Our sample is essentialy complete down to $14.5 \mathrm{mag}$ in $V$. This is roughly the limit for which CoRoT obtains light curves in three colours, fainter stars are only observed in the monochromatic mode, and it is also roughly the limit to which we can detect planets of A stars.

Guiding was performed by placing a fibre bundle on a bright star in the field. The field rotation was monitored by placing additional fibres on typically six stars close to the edge of the fieldof-view. We used the $580 \mathrm{~V}$ grating in the blue arm and the $385 \mathrm{R}$ in the red arm. The spectra cover the range from 3740 to $5810 \AA$ in the blue arm, and 5650 to $8770 \AA$ in the red arm. The resolution is about $R=1300$ for both arms. Each field was observed for 30 to $45 \mathrm{~min}$. To avoid saturation and to facilitate the removal of cosmic rays, we split the observing time spent on each field into three or more exposures.

All calibration frames such as flats, bias-frames, and arcs (using a $\mathrm{CuAr}+\mathrm{FeAr}$-lamp for wavelength-calibration) were taken in the usual way. Bias frames were taken each day in the afternoon with the dome closed and the light switched off. Because the detector is operating at a temperature of $160 \mathrm{~K}$ (Sharp et al. 2006), no dark frames were required. Flats and arcs were taken during the night before the observation of each field. We subtracted the sky-background from each spectrum using the average spectrum of the night-sky taken with the sky-fibres, taking the throughput of each individual fibre into account. We measured the throughput of each individual fibre for each setting always after the fibres were configured, because the throughput changes depending on how the fibre is bent. As usual, the fibres for the first two fields were positioned in the afternoon. We then measured the throughput for each fibre by obtaining spectra during dusk. The same procedure was also performed using the dawn for the last two fields. Using spectra of the blank sky, we 


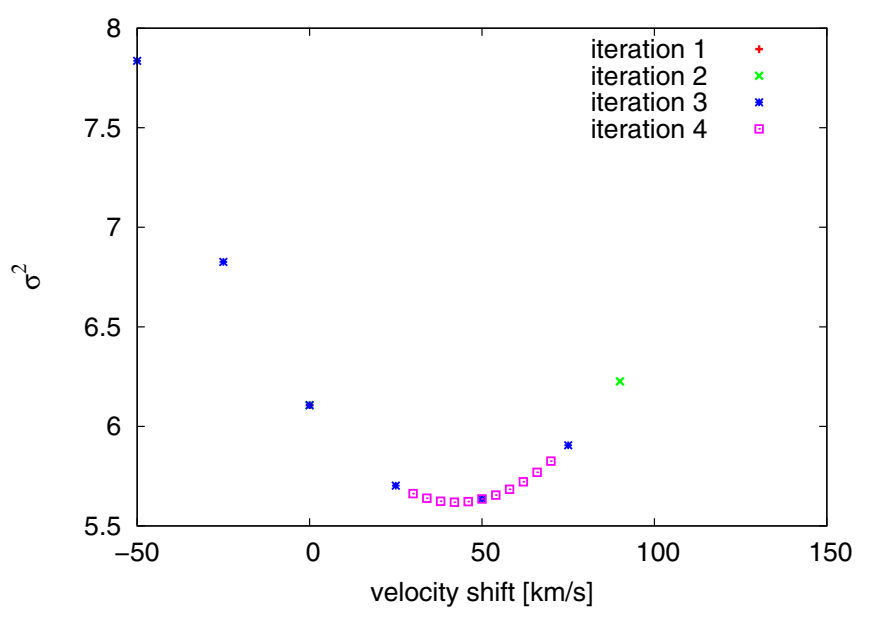

Fig. 3. The spectral types are obtained iteratively by fitting the observed spectra to templates by minimizing $\sigma^{2}$. Shown here is the derived $\sigma^{2}$ vs. the velocty-shift. The best match is obtained at the minimum of $\sigma^{2}$.

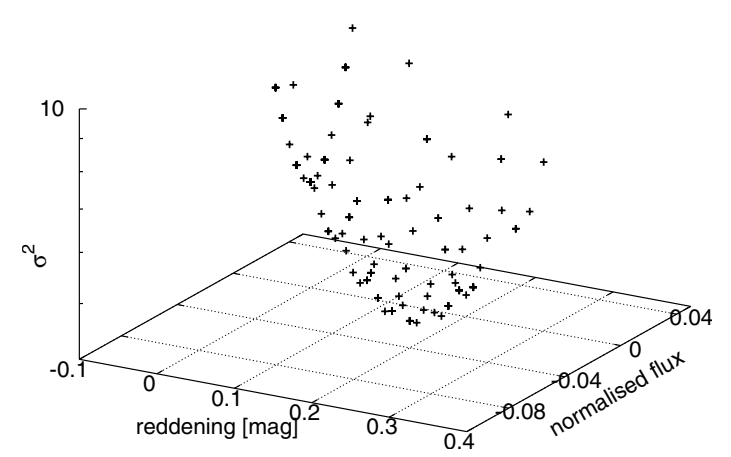

Fig. 4. Same as Fig. 3 but for the flux and the reddening.

measured the throughput for the fields observed at midnight. The sky subtraction is not critical, because we observed only stars brighter than $15.0 \mathrm{mag}$ and the observations were carried out during lunar phases close to new moon. Flux-calibration was finaly made by observing five to ten well-known stars in each field.

\section{Classification of the spectra}

The spectral types of the stars were determined by comparing the observed spectra with templates from a library of spectra automatically. The spectral type of a star is then given by the bestmatching template (see also Gandolfi et al. 2008). As library we used "The Indo-US Library of Coudé Feed Stellar Spectra" (Valdes et al. 2004), which contains a dense grid of spectra of different spectral types and luminosity classes. To fit an observed spectrum to a template, we iteratively adjust the radial velocity, the extinction (Binney \& Merrifield 1998) and the flux of the observed spectrum by minimizing the sum of the differences between the template and the observed spectrum squared $\left(\sigma^{2}\right)$.

Figures 3 and 4 show how the radial-velocity, the flux and the extinction (reddening) were determined by minimising $\sigma^{2}$. For each template we thus derived $\min \left(\sigma^{2}\right)$. Figure 6 shows how $\min \left(\sigma^{2}\right)$ changes for templates of different spectral types. The best-matching template is the one with the smallest $\min \left(\sigma^{2}\right)$, and the spectral type of that template is also the spectral type of the star, A2V in this case. Figure 6 also shows that we can reliably distinguish between dwarf stars and giant stars, but for A and B stars the difference between dwarf stars and sub-giant stars

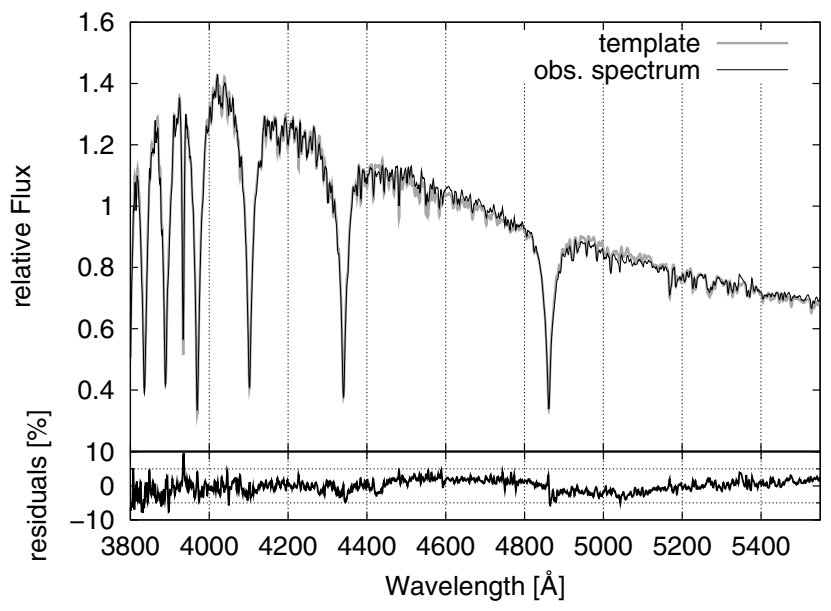

Fig. 5. Spectrum of an A5V-star (black line), together with the template (grey line). The lower panel shows the residuals between the two spectra.

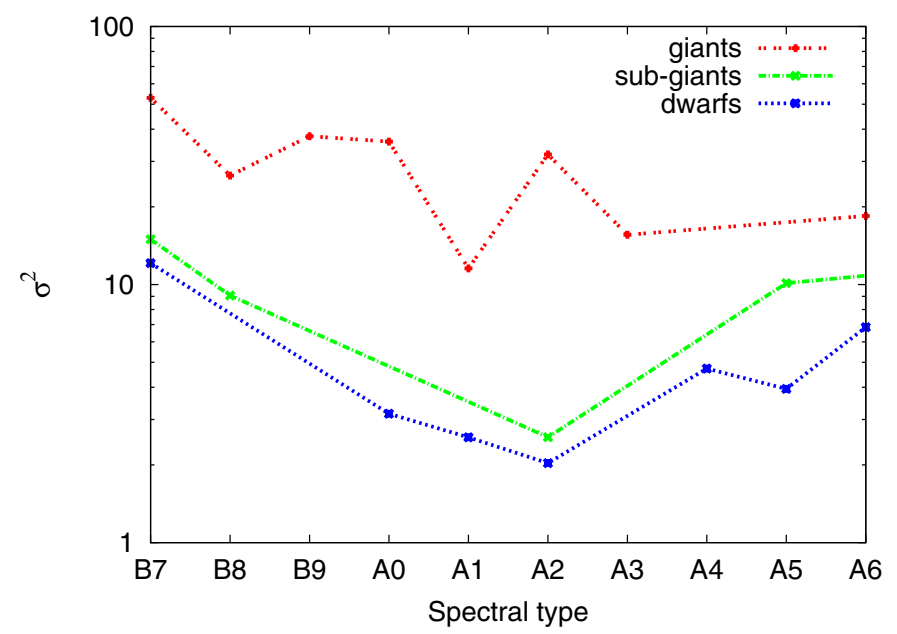

Fig. 6. Shown is $\min \left(\sigma^{2}\right)$ for templates of different spectral types and luminosity classes. $\min \sigma^{2}$ is smallest for the $\mathrm{A} 2 \mathrm{~V}$ template.

is so small that we cannot distinguish between them. Figure 5 shows how well the template matches an observed spectrum. The black line is the observed spectrum of the star, the grey line is the template spectrum.

\section{The accuracy of the method}

Owing to some overlap in the observations for $931 \mathrm{~A}$ and B stars we obtained two or more spectra. By comparing the spectral types derived for the same stars from different spectra, we can therefore determine the error of our method. Figure 7 shows a histogram of the differences in sub-classes between the determination of the spectral type for the same stars. For A and B stars the error is on average $1.61 \pm 0.14$ sub-classes.

There are, however, several outliers were the difference is larger than four sub-classes. Most likely, these stars are intrinsically variable or show chemical peculiarities. Outliers can also be caused by instrumental problems, such as broken or displaced fibres, which cause noisy-spectra. Another instrumental problem that affects a few spectra is a wave-like fringe pattern. In AAOmega starlight enters through a small rectangular prism, which then reflects the light into the fibre (for a detailed description of the instrument, see Saunders et al. 2004; Smith et al. 2004). This prism is normally glued to the fibre entrance 


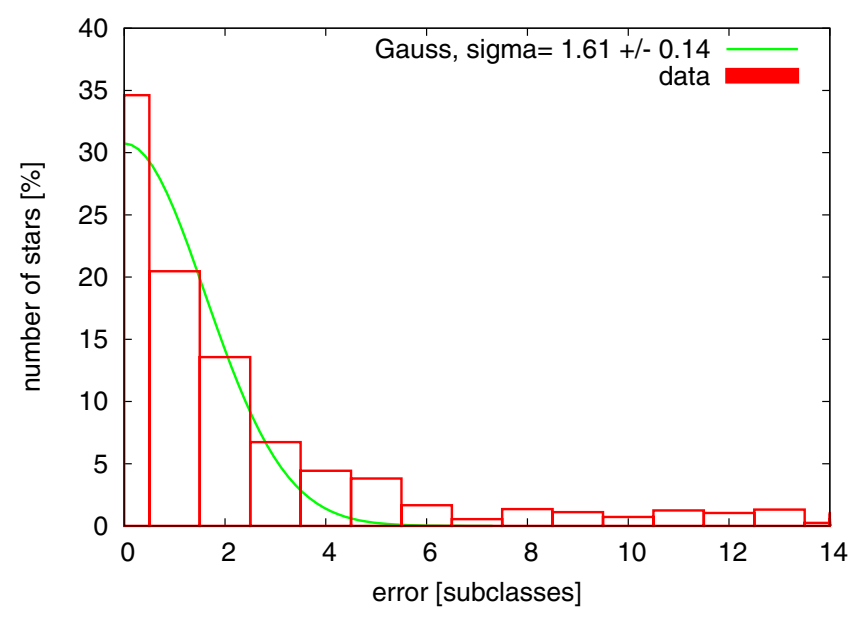

Fig. 7. Error in sub-classes for A and B stars, derived from the difference of the spectral types obtained for stars of which several spectra were taken.

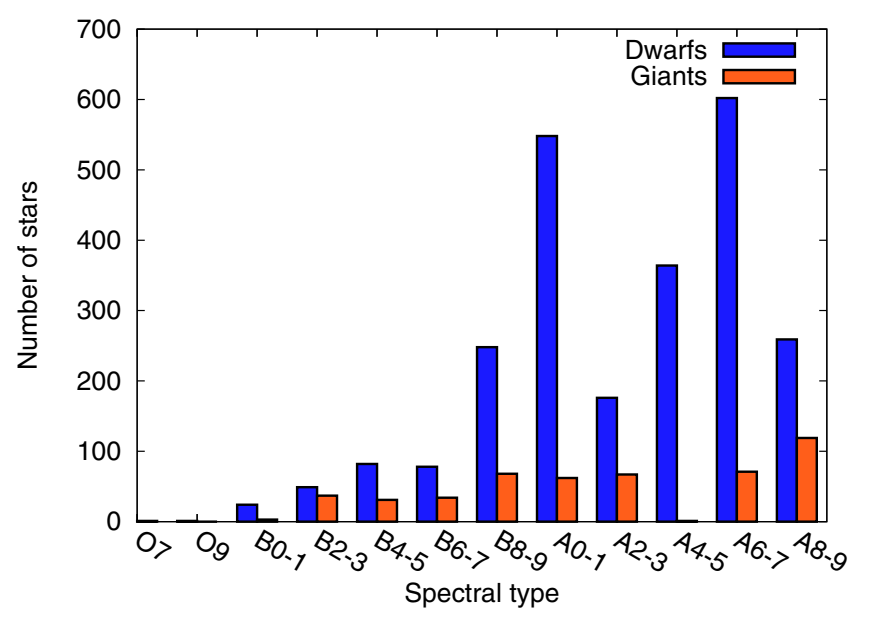

Fig. 8. Distribution of spectral types in our sample. dwarf stars and subgiant stars are combined as "Dwarfs" while giant stars and super-giant stars are combined as "Giants".

but in some cases, the prism is not perfectly glued to the fibre entrance. Then there is a little gap between the fibre and the prism, the resulting "Newtonian rings" then cause the wave-like pattern in these spectra, which reduces the accuracy with which the spectral types can be determined.

However, whenever we had two or more spectra of the same star, we inspected the spectra individually, and disregarded spectra with low signal-to-noise ratio or "Newtonian rings". The error of our spectral classification is therefore certainly smaller than $1.61 \pm 0.14$ sub-classes.

Regarding the accuracy of the classification, it turns out that the spectral classification gives slightly different results when compared with libraries published by different authors (Le Borgne et al. 2003; Jacoby et al. 1984). For this reason we derived the spectral-types by comparing with templates of one library.

\section{The spectral types}

The full spectroscopic survey contains the spectral types of 11466 stars: 2293 A stars, 655 B stars, two O stars and 8516 late-type stars (spectral type $\mathrm{F}$ to $\mathrm{M}$ ). The spectral types of all $\mathrm{O}, \mathrm{B}$, and A stars are listed in Table 1, available at the CDS. All

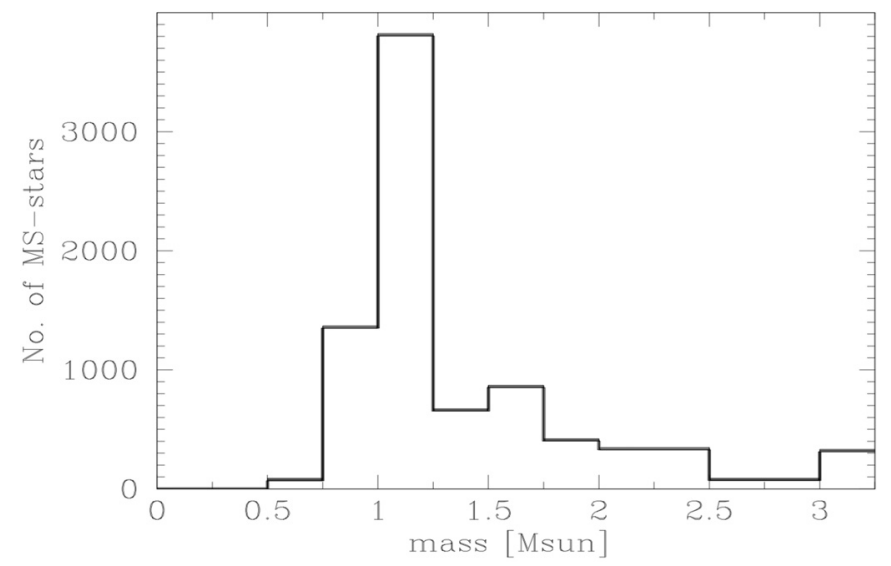

Fig. 9. Mass function of the main-sequence stars in the three CoRoT fields. The last bin contains the number of stars with masses larger than $3 M_{\odot}$.

these stars are in IRa01, LRa01, and LRa02, but not all of them were observed by CoRoT.

The first column in Table 1 gives the CoRoT-ID of the stars where all 1856 stars observed by CoRoT are asterisked in the second column. The third and fourth columns list the coordinates, and the fifth column the brightness of the star in the $V$-band. The visual magnitudes were obtained with the Wide Field Camera filter-system of the Isaac Newton Telescope at Roque de los Muchachos Observatory on La Palma and can be converted into Landolt standards (Landolt 1992), as shown in Deleuil et al. (2009). The last column gives the spectral types that we derived.

\section{Discussion and conclusions}

The distribution of spectral types for early-type stars is shown in Fig. 8. It is interesting to note that giant stars are rare. Of the early-type stars, $82.5 \pm 1.7 \%$ are dwarf stars. At first glance, the lack of stars with a spectral type around A3V seems odd. However, we have to keep in mind that the temperature- and mass range for these spectral types is much smaller than for other spectral types. If we plot the number of stars per mass or temperature interval, the gap disappears. Figure 9 shows the mass-function of main-sequence stars in the IRa01, LRa01, and LRa02 fields. Although the mass function peaks around 1.0 $M_{\odot}$, the main targets of the CoRoT-survey, there is a long slope towards larger masses.

In (Guenther et al., in prep., Paper II), we will show that CoRoT detects one planet-hosting star for $2100 \pm 700$ stars observed. Using this number, we expect to find one transiting planet amongst the A, and B stars in IRa01, LRa01, and LRa02. However, according to Johnson et al. (2010a,b), the frequency of planets orbiting stars in the mass range between 1.5 and $3 M_{\odot}$ is a factor of 2 to 4 higher than for stars of $1.0 M_{\odot}$, which means that we expect to find two to four transiting planets in our sample. By searching for the phase-dependent ellipsoidal variations we will even be able to detect massive, close-in planets and brown dwarfs that are not transiting.

How well do the spectral-types derived photometrically compare with those derived spectroscopically? The photometric classification was taken from the online EXODAT database (Deleuil et al. 2009). It was derived by fitting photometric observations of $B, V, R, J, H, K_{\mathrm{s}}$-colours with spectral energy distribution templates including the reddening. The spectroscopic 


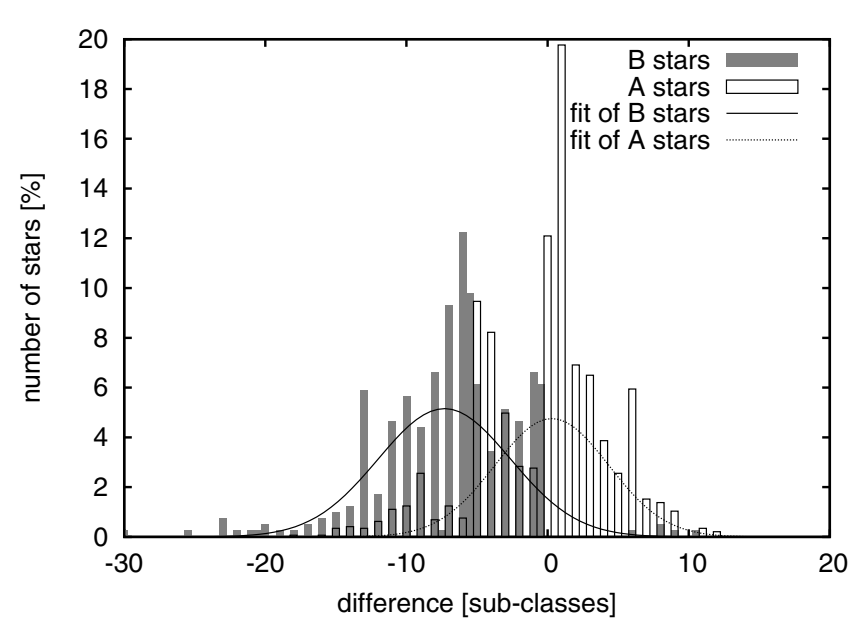

Fig. 10. Differences between spectroscopic and photometric determination of the spectral types. Values lower than zero indicate that the photometric types are later and values higher than zero mean that the photometric types are earlier spectroscopic types. The Gaussian fit roughly characterises the distribution of the samples of spectroscopically classified A and B-type stars.

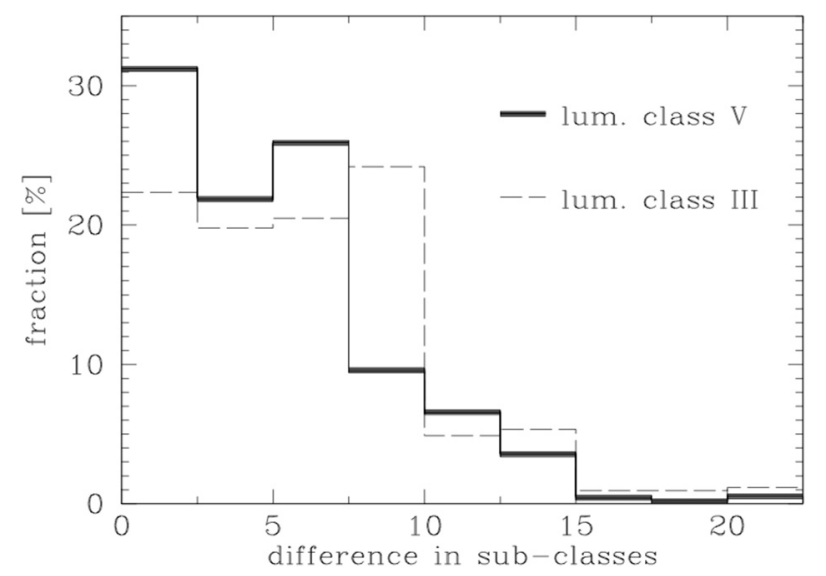

Fig. 11. Absolute differences between the spectroscopic and photometric determination of the spectral types to show the difference for different luminosity classes.

determination was obtained by fitting templates to the observed spectra. Given that the methods used are intrinsically different it is not surprising that the results obtained are also different. As explained by Gray \& Corbally (2009), spectral types are defined by specific spectral features. The analysis of the spectra presented here consequently is the reference against which the photometric classification has to be compared. Figure 10 shows the difference between the photometric and spectroscopic classification of the same stars. Negative values represent stars with a photometric type later ("lower temperature") than the spectroscopic one, whereas positive values represent the stars with earlier ("higher temperature") photometric types. We note that the difference between the photometric and spectroscopic classification is not random but there are systematic differences: The photometric classification of B stars always leads to later spectral types than the spectroscopic one. In contrast to this, A stars peak around identity.

We interpret this result in the way that the photometric method in general produces statistically correct values but the relatively high reddening of the B stars leads to a systematic difference, although the extinction was taken into account in the photometric analysis. Strong local reddening in the observed

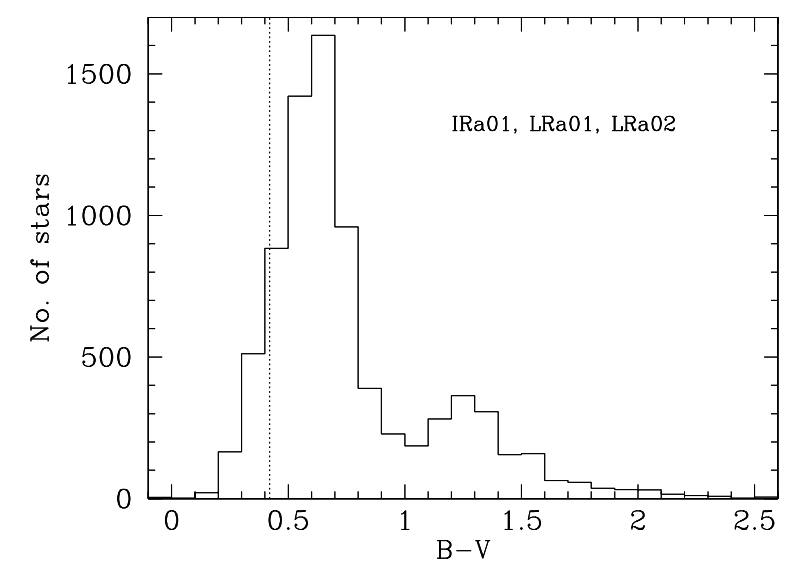

Fig. 12. $B-V$ colours of stars brighter than $m_{V}=14.5$ in the IRa01, $\mathrm{LRa} 01, \mathrm{LRa} 02.70 \%$ of the stars with $B-V<0.42$ (dashed line) are $\mathrm{A}$ and $\mathrm{B}$ stars.

CoRoT-fields is also not surprising since they host known starforming regions e.g. Sh 2-284 (Puga et al. 2009).

Moreover, there are also stars with extremely strong differences in the classification, often caused by blends of more than one star in the fibre. Then the integrated light leads to a combined spectral type. Intrinsic variability of the stars or unresolved spectroscopic binaries might also be responsible for the different spectral types.

Figure 11 shows the comparison between the photometric spectral types and the spectral types from this work in absolute values. For $31 \%$ of the stars the spectral types classified photometrically and spectroscopically are the same. For more than $50 \%$ of the stars the difference is equal or less than five subclasses. We find that $74 \%$ of the stars classified photometrically as A and B stars have a spectral type that is earlier than F3.

Because we now know that the contamination by giant stars is low, and it is possible to identify the A and B stars photometrically, we can extend the survey to all 19983 early-type stars (A and B stars, observed by CoRoT in the long-run fields) listed in EXODAT. Even using the $B-V<0$. 42-criterion (corresponding to an un-reddened F5V star, or an A9V star with $A_{V}=0.18 \mathrm{mag}$ (Binney \& Merrifield 1998)) $70 \%$ of the preselected stars are still real A- and B-type stars (see Fig. 12). The extended sample should lead to the discovery of 10 to 40 planets of early-type stars.

With a sample of planets orbiting intermediate-mass stars we will test the predictions of planet formation theories, and determine the properties of close-in planets orbiting stars with different masses.

By determining the spectral types of early-type stars in these fields, it is now not only possible to carry out an efficient survey of transiting planets orbiting these stars, but it also opens up the possibility for a study of early-type stars in the CoRoT data-base in general.

Acknowledgements. We are grateful to the user support group of AAT for all their help and assistance in preparing and carrying out the observations. We particularly thank Rob Sharp, Fred Watson and Quentin Parker. The authors thank DLR and the German BMBF for the support under grants 50 OW 0204, and 50 OW 0603 as well as DFG for the support of S. Geier through grant HE1356/49-1.

\section{References}

Alibert, Y., Mordasini, C., \& Benz, W. 2011, A\&A, 526, A63 Auvergne, M., Bodin, P., Boisnard, L., et al. 2009, A\&A, 506, 411 
Balona, L. A., Cunha, M. S., Gruberbauer, M., et al. 2011, MNRAS, 413, 2651 Binney, J., \& Merrifield, M. 1998, Galactic Astronomy, ed. J. Binney, \& M. Merrifield (Princeton: Princeton University Press)

Borucki, W. J., Koch, D. G., Basri, G., et al. 2011, ApJ, 736, 19

Bowler, B. P., Johnson, J. A., Marcy, G. W., et al. 2010, ApJ, 709, 396

Cameron, A. C., Guenther, E., Smalley, B., et al. 2010, MNRAS, 407, 507

Carpano, S., Cabrera, J., Alonso, R., et al. 2009, A\&A, 506, 491

Currie, T. 2009, ApJ, 694, L171

Cutri, R. M., Skrutskie, M. F., van Dyk, S., et al. 2003, 2MASS All Sky Catalog of point sources (NASA/IPAC Infrared Science Archive)

Deleuil, M., Moutou, C., Deeg, H. J., et al. 2006, in ESA SP 1306, ed. M. Fridlund, A. Baglin, J. Lochard, \& L. Conroy, 341

Deleuil, M., Meunier, J. C., Moutou, C., et al. 2009, AJ, 138, 649

Gandolfi, D., Alcalá, J. M., Leccia, S., et al. 2008, ApJ, 687, 1303

Gazzano, J.-C., de Laverny, P., Deleuil, M., et al. 2010, A\&A, 523, A91

Gray, R. O., \& Corbally, J. C. 2009, Stellar Spectral Classification, ed. R. O. Gray, \& C. J. Corbally (Princeton: Princeton University Press)

Heber, U. 2009, ARA\&A, 47, 211

Herrero, E., Morales, J. C., Ribas, I., \& Naves, R. 2011, A\&A, 526, L10

Jacoby, G. H., Hunter, D. A., \& Christian, C. A. 1984, ApJS, 56, 257

Johnson, J. A., Aller, K. M., Howard, A. W., \& Crepp, J. R. 2010a, PASP, 122, 905
Johnson, J. A., Bowler, B. P., Howard, A. W., et al. 2010b, ApJ, 721, L153

Kalas, P., Graham, J. R., Chiang, E., et al. 2008, Science, 322, 1345

Kennedy, G. M., \& Kenyon, S. J. 2008, ApJ, 682, 1264

Lagrange, A.-M., Gratadour, D., Chauvin, G., et al. 2009, A\&A, 493, L21

Lammer, H., Odert, P., Leitzinger, M., et al. 2009, A\&A, 506, 399

Landolt, A. U. 1992, AJ, 104, 340

Le Borgne, J., Bruzual, G., Pelló, R., et al. 2003, A\&A, 402, 433

Léger, A., Rouan, D., Schneider, J., et al. 2009, A\&A, 506, 287

Marois, C., Macintosh, B., Barman, T., et al. 2008, Science, 322, 1348

Østensen, R. H., Silvotti, R., Charpinet, S., et al. 2010, MNRAS, 409, 1470

Puga, E., Hony, S., Neiner, C., et al. 2009, A\&A, 503, 107

Sato, B., Izumiura, H., Toyota, E., et al. 2008, PASJ, 60, 539

Saunders, W., Bridges, T., Gillingham, P., et al. 2004, in SPIE Conf. Ser. 5492, ed. A. F. M. Moorwood, \& M. Iye, 389

Sharp, R., Saunders, W., Smith, G., et al. 2006, in SPIE Conf. Ser., 6269, 14

Smith, A. M. S., Anderson, D. R., Skillen, I., Collier Cameron, A., \& Smalley, B. 2011, MNRAS, 416, 2096

Smith, G. A., Saunders, W., Bridges, T., et al. 2004, in SPIE Conf. Ser. 5492, ed. A. F. M. Moorwood, \& M. Iye, 410

Valdes, F., Gupta, R., Rose, J. A., Singh, H. P., \& Bell, D. J. 2004, ApJS, 152, 251

Villaver, E., \& Livio, M. 2009, ApJ, 705, L81 